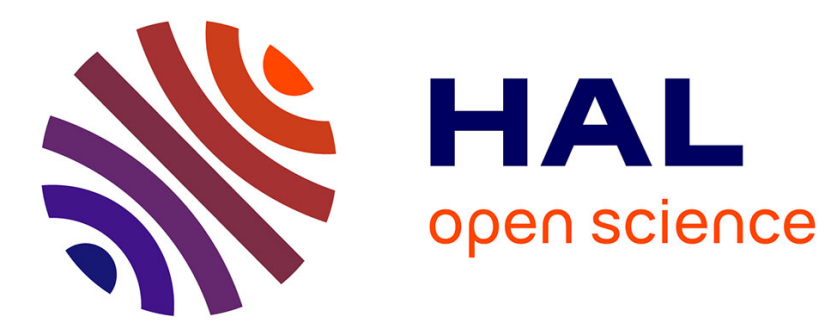

\title{
Étude comparative de divers procédés de transfert d'énergie
}

F. Rioux-Damidau

\section{To cite this version:}

F. Rioux-Damidau. Étude comparative de divers procédés de transfert d'énergie. Revue de Physique Appliquée, 1975, 10 (1), pp.27-34. 10.1051/rphysap:0197500100102700 . jpa-00243875

\section{HAL Id: jpa-00243875 https://hal.science/jpa-00243875}

Submitted on 1 Jan 1975

HAL is a multi-disciplinary open access archive for the deposit and dissemination of scientific research documents, whether they are published or not. The documents may come from teaching and research institutions in France or abroad, or from public or private research centers.
L'archive ouverte pluridisciplinaire HAL, est destinée au dépôt et à la diffusion de documents scientifiques de niveau recherche, publiés ou non, émanant des établissements d'enseignement et de recherche français ou étrangers, des laboratoires publics ou privés. 
Classification

Physics Abstracts

0.660

\title{
ÉTUDE COMPARATIVE DE DIVERS PROCÉDÉS DE TRANSFERT D'ÉNERGIE
}

\author{
F. RIOUX-DAMIDAU
}

Laboratoire de Physique des Plasmas, Université de Paris-Sud, Bâtiment 214, 91405 Orsay, France

(Reçu le 4 juillet 1974, révisé le 20 août 1974)

\begin{abstract}
Résumé. - Pour transférer sur une charge l'énergie stockée dans une bobine, divers moyens peuvent être envisagés. On peut, en particulier, utiliser des interrupteurs à ouverture mécanique en métal normal ou en métal supraconducteur, ou bien un procédé nouveau de transfert inductif à haut rendement placé entre deux interrupteurs ou encore des interrupteurs à transition supraconducteur-conducteur normal. Les performances de ces divers systèmes dépendent du temps de transfert $\tau$ et de la durée $\theta$ du stockage ; elles sont comparées et, pour chaque couple $(\theta, \tau)$, le système le mieux adapté est précisé. On montre en particulier que les interrupteurs supraconducteurs à transition sont moins efficaces que les mécaniques.
\end{abstract}

\begin{abstract}
In order to transfer energy from a storage coil to a load, various devices can be used. These include mechanical breakers made of normal or superconductor metal, and a new process in which a high efficiency inductive transfer device is placed between two switches, either normal or superconducting or superconductor-to-normal transition switch. The choice is governed by the transfer time $\tau$ and the storage time $\theta$. The various devices are compared and the most appropriate one is derived for given values of the parameters $(\theta, \tau)$. In particular, the superconducting transition switches have been found to be less efficient than the superconducting displacement switches.
\end{abstract}

1. Introduction. - Les sources d'énergie électrique impulsionnelles indispensables à de nombreuses applications sont souvent constituées par des bancs de condensateurs. Cependant, lorsqu'on désire atteindre de hauts niveaux d'énergie (supérieurs à quelques MJ), cette technique devient inadaptée. On préfère alors stocker l'énergie sous forme magnétique dans une bobine en métal normal ou supraconducteur et placer en série avec cette bobine un interrupteur convenable capable de transférer en un temps court l'énergie sur une charge branchée à ses bornes. Le problème de la réalisation de cet interrupteur est en général assez difficile car on lui demande des performances élevées. Pendant la durée du stockage, il est traversé par la totalité du courant. Au cours du transfert, il est soumis à une tension élevée. D'autre part, pour assurer son fonctionnement, il est indiqué de mettre en œuvre aussi peu d'énergie que possible. En particulier, il faut que l'énergie dépensée à la fois pour la commande et par effet Joule soit nettement inférieure à l'énergie transférée. Enfin, il est bon aussi que le volume de l'interrupteur soit raisonnable.

Dans un précédent article [1], nous avons étudié les performances générales d'interrupteurs à rupture mécanique simples dans lesquels un morceau du circuit conducteur était remplacé rapidement par de l'isolant. Après avoir précisé ses performances dans le cas d'une utilisation pratique, nous envisagerons pour certaines applications de mettre plusieurs interrupteurs en parallèle. Nous étudierons ensuite divers autres moyens propres à effectuer des transferts d'énergie. Nous examinerons l'intérêt de l'emploi des métaux supraconducteurs sous la forme d'interrupteurs à ouverture mécanique par déplacement d'un métal supraconducteur ou d'interrupteurs à transition supraconducteur-conducteur [2]. Par ailleurs, nous discuterons la possibilité d'utiliser un procédé nouveau de transfert inductif à haut rendement [3] placé entre deux interrupteurs. Nous pourrons ainsi préciser les performances relatives de ces divers procédés de transfert d'énergie.

2. Interrupteur à rupture mécanique de conducteur. 2.1 Performances D'UN INTERRUPTEUR UNIQUE. Un tel interrupteur a déjà été étudié [1]. Son fonctionnement est rappelé sur la figure 1 . Un courant de densité $i$ circule initialement dans l'interrupteur pendant la phase de fermeture de durée $\theta$ (que nous appelions $T_{0}$ dans [1]). A la fin de cette période, le transfert du courant sur la charge s'effectue en un temps très court ; la tension $U(t)$ supportable ensuite par l'interrupteur croît progressivement avec le temps. 
Elle atteint sa valeur maximale $U_{\mathrm{M}}$ au bout d'un temps de réisolement $T$ lorsque le morceau de conducteur découpé a parcouru une distance égale à $l / 2, l$ étant la dimension caractéristique de la découpe. (On a

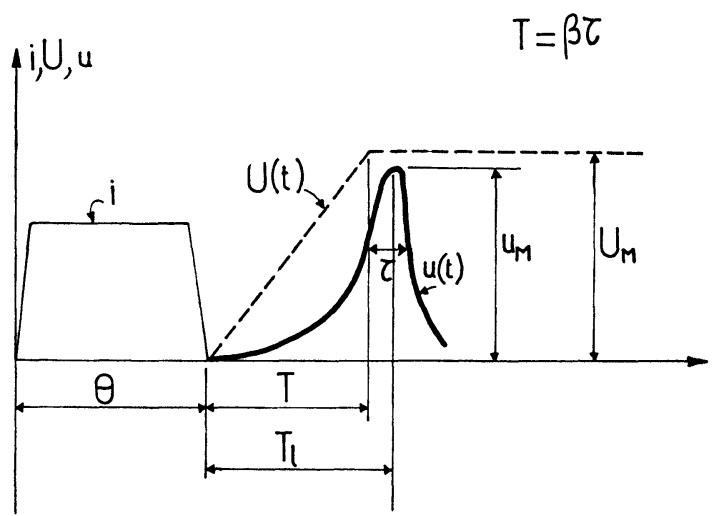

Fig. 1. - Variation en fonction du temps de la densité de courant $i$ et de la tension $u$ pour l'interrupteur. En pointillé, nous avons représenté la tension maximale admissible $U$.

$U_{\mathrm{M}}=E l$ où $E$ est le champ électrique limite admissible par l'isolant.) Nous avons montré que l'impédance de la charge devait rester faible au cours du transfert du courant et croître ensuite très vite lors de la phase du transfert d'énergie pour que la récupération de cette dernière puisse s'effectuer rapidement. Dans ces conditions, l'énergie requise pour le transfert du courant est faible ; elle peut par exemple être fournie par un condensateur annexe ou directement par l'arc créé lors de l'ouverture. Aussi, nous supposons que l'impédance de la charge est petite pendant un temps $T_{l}$ dit temps de latence puis prend une valeur élevée pendant le temps $\tau$ (si la charge effective est fixe on peut, en mettant par exemple un fil explosant en parallèle, réaliser une charge apparente ayant ces caractéristiques). On pose alors $T_{l}=\beta_{0} \tau$ où le paramètre $\beta_{0}$, caractéristique de la charge, est en général petit, de l'ordre de quelques unités; nous adopterons comme valeur typique $\beta_{0}=5$. L'interrupteur est donc soumis à une tension $u(t)$ qui présente une pointe d'amplitude $u_{\mathrm{M}}$ et de durée $\tau$ au bout du temps $T_{l}$. Cette tension doit vérifier $u<U$. Lorsque la charge est bien adaptée, comme nous le supposerons désormais, on a $u_{\mathrm{M}} \simeq U_{\mathrm{M}}$ et $T_{l} \simeq T$; nous écrirons alors $T=\beta \tau$ avec $\beta \simeq \beta_{0}$. L'énergie transférée par

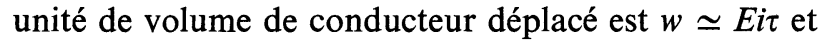
les pertes relatives sont $\eta=\frac{w_{\mathrm{J}}+w_{\mathrm{R}}}{w}$ où $w_{\mathrm{J}}$ est la densité d'énergie Joule dépensée dans le conducteur à rompre pendant le temps $\theta$ et $w_{\mathrm{R}}$ la densité d'énergie fournie pour déplacer le conducteur. On posera : $\left(w_{\mathrm{R}}=w_{\mathrm{c}}\right.$, où $w_{\mathrm{c}}$ est la densité d'énergie cinétique du morceau de conducteur déplacé au cours de la rupture).

Notre étude [1] avait permis en particulier de préciser pour chaque couple de valeurs $\theta$ et $\tau$ que l'on s'imposait les meilleures valeurs des paramètres physiques à choisir et les performances $\eta$ et $w$ qui en résultaient. Nous avions montré ainsi que le cuivre et l'aluminium conduisaient à des performances équivalentes et qu'il était toujours préférable d'avoir un isolant à valeur de $E$ très élevée (tout restant égal par ailleurs, $w$ est proportionnel à $E$ et $\eta$ à $1 / E$ ). Désormais, nous allons supposer que $E=3 \times 10^{7} \mathrm{~V} / \mathrm{m}$ et que le conducteur est du cuivre (résistivité $\rho_{0}$, capacité calorifique $c$, masse spécifique $\bar{\omega}$, coefficient de diffusion thermique $k$. On pose également: $w_{0}=\bar{\omega} \mathrm{c} \theta_{0}$ avec $\theta_{0}=300 \mathrm{~K}$ ).

Pour caractériser la possibilité pratique de réaliser un interrupteur, il est intéressant de connaître la valeur maximale de la densité d'énergie transférable $w_{\mathrm{Q}}$ associée aux valeurs $(\theta, \tau)$ lorsqu'on impose $\eta \leqslant \eta_{\mathrm{M}}$. En effet, le volume $W / w_{\mathrm{Q}}$ de matériau à rompre, nécessaire pour transférer une énergie donnée $W$ avec des pertes relatives au plus égales à $\eta_{\mathrm{M}}$, est une donnée très importante dans une construction.

$\mathrm{La}$ détermination des caractéristiques de fonctionnement répondant à ces conditions est reportée en appendice. Nous en avons déduit le diagramme de la figure 2 qui rassemble l'essentiel des résultats. Sur ce diagramme en $\theta$ et $\tau$ sont indiquées les courbes $w_{\mathrm{Q}}(\theta, \tau)=$ Cte. Ces courbes se composant de trois segments de droite, nous avons distingué trois zones $\mathrm{B}, \mathrm{C}$ et $\mathrm{D}$. Dans cette dernière zone, $w_{\mathrm{Q}}$ ne dépend que de la nature de l'isolant et de celle du conducteur. Dans $\mathrm{B}, w_{\mathrm{Q}}$ dépend en outre de $\eta_{\mathrm{M}}$ et dans $\mathrm{C}$ de $l$. De plus, $\eta_{\mathrm{P}}=\eta_{\mathrm{M}}$ dans $\mathrm{B}$ et $\eta_{\mathrm{P}} \leqslant \eta_{\mathrm{M}}$ dans $\mathrm{C}$ et $\mathrm{D}$. Ainsi, la position dans ce diagramme du point $P$ de coordonnées $(\theta, \tau)$ donne par simple lecture la valeur de $w_{\mathrm{Q}}$. Cependant, tous les points $P$ ne correspondent pas à un fonctionnement possible dans les conditions demandées. Pour être valables, ils doivent être situés

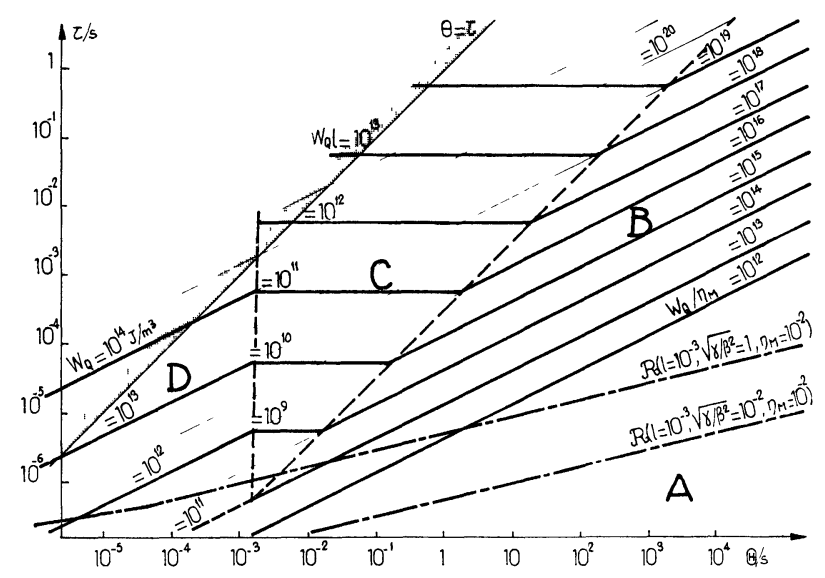

FIG. 2. - Valeur de la densité maximum d'énergie transférable $w_{\mathrm{Q}}$ en fonction de $\theta$ et $\tau$ lorsque les pertes relatives $\eta$ vérifient $\eta \leqslant \eta_{\mathrm{M}}$. Trois types de courbes apparaissent: $w_{\mathrm{Q}} / \eta_{\mathrm{M}}=$ Cte dans la partie $\mathrm{B}, w_{\mathrm{Q}} l=\mathrm{Cte}$ dans la partie $\mathrm{C}$ et $w_{\mathrm{Q}}=\mathrm{Cte}$ dans la partie D ; elles ont été tracées en pointillés. Nous avons tracé en traits pleins les courbes $w_{\mathrm{Q}}=$ Cte correspondant à $\eta_{\mathrm{M}}=10^{-2}$ et $l=1 \mathrm{~mm}$. Dans la partie A située sous la courbe $\mathcal{R}$, il n'existe pas d'interrupteur ayant un rendement convenable. Ce diagramme a été tracé pour du cuivre et un isolant pour lequel $E=3 \times 10^{7} \mathrm{~V} / \mathrm{m}$ 
en dehors de la zone A limitée par la courbe $\mathcal{R}$. La position de $\mathcal{R}$ dépend de $l, \sqrt{\gamma / \beta^{2}}$ et $\eta_{\mathbf{M}}$. Comme la durée du stockage $\theta$ est toujours supérieure à $\beta \tau$ nous avons aussi porté la limite $\theta=\beta \tau$.

Nous constatons finalement que les valeurs limites atteignables avec un interrupteur simple en ce qui concerne $\theta$ et $\tau$ sont données par la courbe $\mathcal{R}$ correspondant aux valeurs extrémales $l_{\mathrm{e}}, \beta_{\mathrm{e}}, \gamma_{\mathrm{e}}$ et $\eta_{\mathrm{e}}$ des paramètres (minimales pour $l$ et $\gamma$, maximales pour $\beta$ et $\eta$ ) : nous l'appellerons $\mathcal{R}_{\mathrm{e}}$. On peut cependant se demander si, en plaçant plusieurs interrupteurs en parallèle pour former un interrupteur multiple, on ne pourrait pas en élargir le domaine d'utilisation. C'est pourquoi nous allons maintenant considérer une telle association.

2.2 Performances D'UN ENSEMble DE DeUX INTERRUPTEURS EN PARALLÈLE. - L'interrupteur global, pour lequel le temps de passage initial du courant est $\theta$ et le temps de transfert d'énergie $\tau$, est formé par les deux interrupteurs en parallèle $J_{a}$ et $J_{b}$ (voir Fig. 3). Le courant circule tout d'abord dans $J_{a}$ pendant le temps $\theta$ au bout duquel on ouvre $J_{a}$ en transférant le courant sur $\mathfrak{J}_{b}$. Le réisolement de $\mathfrak{J}_{\mathbf{a}}$ s'effectue en un temps $T_{\mathrm{a}}$ au bout duquel on ouvre $J_{\mathrm{b}}$ (on a donc $\theta_{\mathrm{b}} \simeq T_{\mathrm{a}}$ ), le temps de réisolement de $J_{\mathrm{b}}$ étant $T_{\mathrm{b}}$. Le transfert d'énergie sur la charge applique alors une pointe de tension de durée $\tau$ sur $J_{a}$ et $J_{b}$ et l'on a :

$$
T_{\mathrm{a}}=\beta_{\mathrm{a}} \tau \quad T_{\mathrm{b}}=\beta_{\mathrm{b}} \tau .
$$

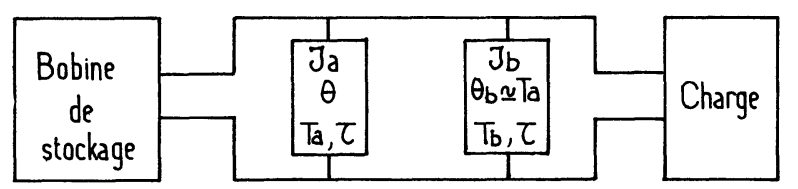

FIG. 3. - Interrupteur double formé de $\mathfrak{J}_{\mathrm{a}}$ et $\mathfrak{J}_{\mathrm{b}}$ en parallèle.

Il faut remarquer que si la charge est convenablement adaptée à l'interrupteur secondaire, c'est-à-dire si $T_{\mathrm{b}}$ est de l'ordre de grandeur du temps de latence de la charge, $\beta_{\mathrm{b}}$ est caractéristique de cette dernière et est petit. En ce qui concerne $\mathfrak{J}_{a}$, le rôle de la charge est joué par $\mathfrak{J}_{b}$. Or « le temps de latence» de $\mathfrak{J}_{b}$, c'està-dire $\theta_{\mathrm{b}}$, n'est pas lié de façon stricte à $\tau$ comme pour une impédance variable : $\beta_{\mathrm{a}}$ peut prendre des valeurs appréciables. On peut donc réaliser $\beta_{\mathrm{a}} \gg \beta_{\mathrm{b}}$.

Par ailleurs, le temps $\tau$ étant le même pour les deux interrupteurs $\mathfrak{J}_{\mathbf{a}}$ et $\mathfrak{J}_{\mathrm{b}}$, leurs points représentatifs $P_{\mathrm{a}}$ et $P_{\mathrm{b}}$ sont situés sur une même horizontale, $P_{\mathrm{a}}$ étant à droite de $P_{\mathrm{b}}$ (voir Fig. 4): la densité d'énergie transférable par $J_{b}$ est toujours supérieure à celle transférable par $J_{\mathbf{a}} . J_{\mathbf{a}}$ sera donc plus gros que $J_{b}$ puisque l'énergie $U I \tau$ transférée par ces deux interrupteurs est la même.

Si $P_{\mathrm{a}}$ est au-dessus de $\mathcal{R}_{\mathrm{e}}$, il existe en fait un interrupteur simple $J_{0}$ dont le point représentatif est $P_{\mathrm{a}}$, dont les caractéristiques sont $\theta, \tau$ et $\beta \sim \beta_{\mathrm{b}}$ et qui peut donc remplacer l'interrupteur global $J_{a}+J_{b}$. La densité d'énergie transférable par chacun des deux inter-

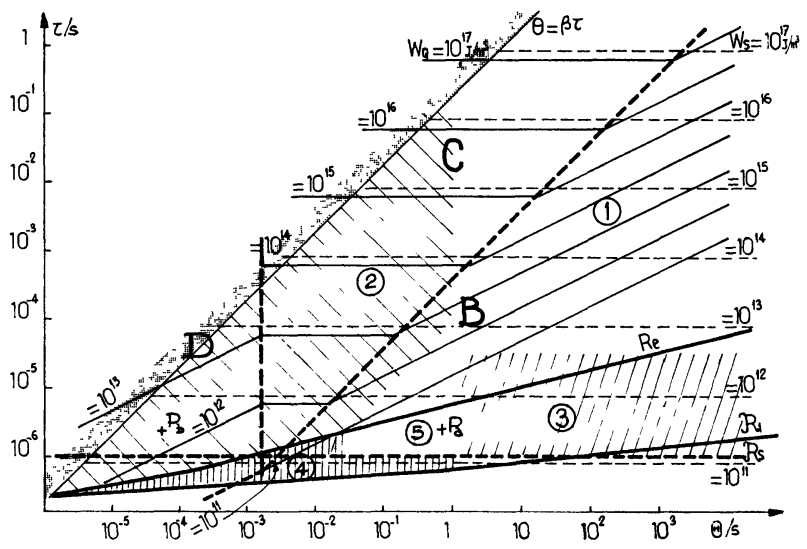

Fig. 4. - Caractéristiques des disjoncteurs mécaniques. Courbes $w_{\mathrm{Q}}(\theta, \tau)=$ Cte pour un interrupteur en cuivre $(l=1 \mathrm{~mm}$, $\left.\eta=10^{-2}\right)$ et $w_{\mathrm{s}}(\theta, \tau)=$ Cte pour un interrupteur en supraconducteur. Nous avons indiqué le meilleur interrupteur mécanique à utiliser pour $\theta$ et $\tau$ donnés : dans la zone (1), c'est un interrupteur en supraconducteur unique, dans la zone (2) un interrupteur en cuivre, dans la zone (3) un interrupteur en supraconducteur $J_{\mathfrak{a}}$ en parallèle avec un interrupteur $J_{\mathfrak{b}}$ en cuivre et dans la zone (4) deux interrupteurs $J_{a}$ et $J_{b}$ en cuivre en parallèle. Dans (5), différents choix sont possibles. Les courbes limites d'existence des différents disjoncteurs sont : $\mathcal{R}_{\mathrm{e}}$ pour un interrupteur unique en cuivre, $\mathcal{R}_{\mathrm{s}}$ pour un interrupteur unique en supraconducteur et $\mathcal{R}_{\mathrm{a}}$ pour deux interrupteurs en cuivre en parallèle.

rupteurs $J_{a}$ et $J_{0}$ qui sont représentés par le même point est la même si les valeurs de $l, \eta_{M}$ et $\gamma$ sont identiques. Nous voyons que dans ce cas, l'ensemble plus complexe $J_{\mathbf{a}}+J_{\mathbf{b}}$ n'apporte $a$ priori aucun avantage nouveau.

Nous avons montré au paragraphe précédent que $\mathcal{R}_{\mathrm{e}}$ représentait la limite d'existence des interrupteurs simples où $\beta$ était déterminé par la charge. Ici, $\mathcal{R}_{\mathrm{e}}$ représente donc la limite d'existence des interrupteurs $\mathfrak{J}_{\mathrm{b}}$ puisque $\beta_{\mathrm{b}}$ est lié à la charge. Comme $\beta_{\mathrm{a}} \gg \beta_{\mathrm{b}}$, le domaine d'existence de $J_{\mathrm{a}}$ est plus grand. $\beta_{\mathrm{a}}$ étant donné par le rapport $T_{\mathrm{a}} / \tau=\theta_{\mathrm{b}} / \tau$, on obtient sa plus grande valeur correspondant à un $\tau$ donné lorsque $P_{\mathrm{b}}$ est sur $\mathcal{R}_{\mathrm{e}}$. Nous avons ainsi déduit la courbe limite $\mathcal{R}_{\mathrm{a}}$ d'existence de $\mathfrak{J}_{\mathrm{a}}$ en utilisant les éq. (34), (36) et (37) de [1].

2.3 ConClusion. - Lorsqu'on désire transférer en un temps $\tau$ sur une charge d'utilisation l'énergie qui a été stockée durant un temps $\theta$ dans une bobine à l'aide d'un interrupteur simple ou double à déplacement de conducteur, les caractéristiques de ce dernier s'obtiennent facilement en portant le point $P_{a}$ de coordonnées $(\theta, \tau)$ sur la figure 2 .

Si $P_{a}$ est au-dessus de $\mathcal{R}_{\mathrm{e}}$, un interrupteur unique est ce qui convient le mieux.

Si $\mathrm{P}_{\mathrm{a}}$ est compris entre $\mathcal{R}_{\mathrm{e}}$ et $\mathcal{R}_{\mathrm{a}}$, le transfert sera effectué à l'aide de deux interrupteurs en parallèle.

Nous n'envisagerons pas la mise en parallèle de plus de deux interrupteurs car elle ne semble pas compétitive avec les procédés que nous allons décrire ci-dessous. 
Finalement, nous voyons que les temps $\tau$ les plus courts que l'on peut atteindre sont un peu inférieurs à la microseconde.

3. Comparaison des interrupteurs à déplacement de conducteur et de supraconducteur. - Les possibilités des interrupteurs à conducteur normal sont limitées par l'effet Joule en ce qui concerne les temps de stockage $\theta$ longs. Aussi, pour pallier à cette difficulté, on peut envisager de leur substituer des métaux supraconducteurs que l'on couperait ou tout au moins que l'on déplacerait (par exemple en écartant deux électrodes). Dans ce cas, il n'y aurait aucune perte Joule pendant le temps de passage initial du courant et la dépense énergétique serait due uniquement au déplacement du supraconducteur. Si nous adoptons cette solution, les pertes relatives valent :

$$
\eta=\eta_{\mathrm{R}}=\frac{w_{\mathrm{R}}}{w_{\mathrm{s}}}
$$

où la densité d'énergie de rupture peut toujours s'écrire sous la forme $w_{\mathrm{R}}=\frac{1}{2} \gamma \bar{\omega} v^{2}, \gamma$ étant le rapport entre l'énergie de rupture dépensée et l'énergie cinétique du morceau découpé lorsqu'il s'est déplacé de la distance $l / 2=\beta \tau$. La densité d'énergie transférée $w_{\mathrm{s}}$ s'écrit encore :

$$
w_{\mathrm{s}}=E i \tau
$$

où $i$ est la densité de courant dans le supraconducteur avant l'ouverture de l'interrupteur. Finalement on a donc :

$$
\eta=\frac{\bar{\omega}}{8 E i} \cdot \frac{\gamma}{\beta^{2}} \cdot \frac{l^{2}}{\tau^{3}} .
$$

Comparons alors les performances des interrupteurs à déplacement de conducteur et de supraconducteur. Nous supposerons dans les deux cas utiliser des isolants de même pouvoir isolant. D'autre part, nous admettrons que le supraconducteur est parcouru par la densité de courant maximum admissible que nous prenons égale à $4 \times 10^{9} \mathrm{~A} / \mathrm{m}^{2}$ [2]. Les pertes relatives qui sont inversement proportionnelles à $\tau^{3}$ (cf.(3)) ne deviennent alors importantes que pour $\tau$ très petit. Nous avons tracé sur la figure 4 la droite $\mathcal{R}_{\mathrm{s}}$ qui délimite les possibilités de fonctionnement avec des pertes inférieures à $10^{-2}$ des interrupteurs à déplacement de supraconducteur et dont l'équation est donnée par (3). Nous avons aussi porté sur cette même figure les droites $w_{\mathrm{s}}=$ Cte et les courbes $w_{\mathrm{Q}}=$ Cte en admettant dans ce dernier cas que $l=1 \mathrm{~mm}$ dans la région $\mathrm{C}$. Nous allons ainsi pouvoir comparer les densités d'énergie transférables par les deux types d'interrupteurs et en déduire leurs domaines d'utilisation respectifs.

Tout d'abord, nous remarquons que, dans la zone $C$, $w_{\mathrm{s}}$ et $w_{\mathrm{Q}}$ sont très équivalents. La densité de courant admissible par le supraconducteur est en effet du même ordre que celle admissible par le cuivre lorsque la chaleur dégagée par effet Joule dans la partie du conducteur qui doit être cassée diffuse vers des régions froides, cette partie prenant très rapidement une température stationnaire. Dans cette région $\mathrm{C}$, le choix entre les deux types d'interrupteurs sera donc dicté par d'autres considérations que la valeur de la densité d'énergie transférable. Il en sera de même dans la partie de la zone $B$ située au voisinage de la zone $C$.

Dans la partie de la zone $\mathrm{B}$ où $w_{\mathrm{s}} \gg w_{\mathrm{Q}}$, on choisira naturellement le supraconducteur (zone (1)).

Dans la zone $\mathrm{D}, w_{\mathrm{s}} \lesssim w_{\mathrm{Q}}$. A priori, le cuivre sera préférable pour des questions pratiques.

Pour finir de préciser les domaines d'utilisation des deux types d'interrupteurs, notons que le stockage initial de l'énergie se fait avec des bobines en cuivre lorsque $\theta$ ne dépasse pas la seconde en ordre de grandeur et avec des bobines supraconductrices dans le cas contraire. Nous distinguerons alors les cas suivants :

- Si $\theta \lesssim 1 \mathrm{~s}$ et si le point $\mathrm{P}(\theta, \tau)$ est dans les zones $\mathrm{C}$ ou $\mathrm{D}$ ou même dans la zone $\mathrm{B}$ à la limite de la zone $\mathrm{C}$, ce qui convient le mieux est un interrupteur à déplacement de conducteur normal (unique ou formé par la mise en parallèle de deux d'entre eux) (zones (2) et (4)).

- Si l'énergie a été stockée initialement dans une bobine supraconductrice, c'est-à-dire si $\theta \gtrsim 1 \mathrm{~s}$, on utilisera un interrupteur à déplacement de supraconducteur. Cependant, il faut remarquer que la vitesse de déplacement du morceau remplacé par de l'isolant est $v=l / 2 \beta \tau$. Pour l'interrupteur à supraconducteur simple considéré (zone (1)), $\beta$ est déterminé par la charge et vaut quelques unités. Si nous admettons que la limite des vitesses réalisables avec un dispositif mécanique simple est de $3 \mathrm{~m} / \mathrm{s}$, nous en déduisons que $\tau \gtrsim 3 \times 10^{-5} \mathrm{~s}$. Aussi, lorsqu'on désire obtenir un temps de transfert d'énergie plus court, on préférera associer un interrupteur secondaire en cuivre à l'interrupteur supraconducteur (zone (3)) ; pour ce dernier, qui sera donc un interrupteur primaire, $\beta_{\mathrm{p}}$ sera élevé et la vitesse à mettre en jeu petite. Si on impose que $v$ soit égal à $3 \mathrm{~m} / \mathrm{s}$, on aura alors dans tous les cas $\theta_{\mathrm{s}}=\beta_{\mathrm{p}} \tau=1,5 \times 10^{-4} \mathrm{~s}$.

- Enfin, si le stockage se fait avec une bobine en cuivre et si $\mathrm{P}$ est situé dans la partie de la zone $\mathrm{B}$ où $w_{\mathrm{s}}>w_{\mathrm{Q}}$ c'est-à-dire lorsque $10^{-1} \lesssim \theta \lesssim 1 \mathrm{~s}$ et $10^{-6} \lesssim \tau \lesssim \mathrm{qqs} 10^{-5} \mathrm{~s}$, les considérations énoncées jusqu'ici nous conduiraient à associer un interrupteur primaire supraconducteur et un interrupteur secondaire en cuivre. Pour des questions pratiques, on pourrait cependant préférer que l'interrupteur primaire soit aussi en cuivre ou, lorsque c'est possible, qu'il y ait un seul interrupteur en cuivre. En fait, nous allons voir maintenant qu'une autre technique très différente peut être utilisée dans ces domaines.

4. Utilisation des systèmes de transfert inductif à haut rendement (T. I. H. R.). - Lorsque la durée $\theta$ du stockage d'énergie est assez longue et le transfert 
d'énergie court (c'est-à-dire en particulier lorsque $P(\theta, \tau)$ est dans la zone (3) de la Fig. 4), la densité d'énergie transférable par l'interrupteur primaire devient faible. Ce dernier peut alors atteindre des dimensions importantes. Aussi, une solution un peu plus complexe peut être adoptée [3]. Le schéma de principe en est représenté figure 5. L'interrupteur $\mathfrak{J}_{1}$ étant fermé, la bobine $B_{1}$ stocke l'énergie initiale pendant le temps $\theta$. A la fin de cette période, on ferme $\mathrm{C}$ et on ouvre en un temps $\tau_{1}$ l'interrupteur $\mathfrak{J}_{1}$. Puis, à l'aide d'un système assez compliqué $\mathrm{g}$ de commutations successives on transfère en un temps $\theta$, avec un rendement élevé, l'énergie de la bobine $B_{1}$ vers la bobine $B_{2}$ distincte spatialement de $B_{1}$. Enfin, on transfère l'énergie de $B_{2}$ vers l'utilisation en ouvrant l'interrupteur $\mathfrak{J}_{2}$.

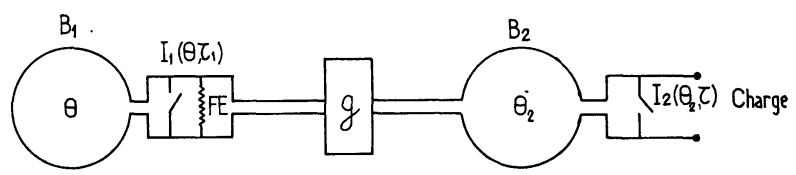

FIG. 5. - Schéma de principe du procédé de transfert inductif à haut rendement (T. I. H. R.). L'énergie stockée dans la bobine $B_{1}$ pendant le temps $\theta$ est transférée dans la bobine $B_{2}$ à l'aide de l'interrupteur $\mathfrak{J}_{1}$, du fil explosé $\mathrm{FE}$ et du système spécial $\mathrm{g}$ en un temps $\tau_{1} \lesssim \theta_{2}$. Puis l'énergie stockée pendant le temps $\theta_{2}$ dans la bobine $\mathrm{B}_{2}$ est transférée par l'intermédiaire de l'interrupteur $\mathfrak{J}_{2}$ sur la charge d'utilisation.

Ce système appelle les remarques suivantes :

- En général, on place aux bornes de $J_{1}$ un fil explosant FE qui, étant donné la nature de $\mathrm{g}$, n'a qu'une énergie très faible à absorber. Les paramètres caractéristiques de $J_{1}$ sont alors $\theta$ qui est imposé, $\tau_{1}$ qui est tel que $\tau_{1} \lesssim \theta_{2}$ et $\beta_{1}=\beta$ (fil). $\beta_{1}$ est donc encore de l'ordre de 5 . La tension vue par $J_{1}$ lors du transfert d'énergie sur la charge d'utilisation n'est pas $U_{\mathrm{M}}$ mais seulement $U_{\mathrm{M}} \frac{\tau_{1}}{\tau}$.

- L'interrupteur $\mathfrak{J}_{2}$ doit certes supporter toute la tension $U_{\mathrm{M}}$ mais le temps $\theta_{2}$ de passage initial du courant est fortement réduit par rapport au temps de stockage initial de l'énergie. Ses paramètres sont $\theta_{2}, \tau$ et $\beta$ où $\beta$ est caractéristique de l'utilisation.

Le choix de $\theta_{2}$ et $\tau_{1}$ est a priori assez libre; il faut simplement que l'on vérifie $\tau_{1} \leqslant \theta_{2}$. Cependant il semble raisonnable d'équilibrer les performances demandées à chacun des deux interrupteurs. C'est pourquoi nous imposerons $\theta_{2}=\tau_{1}$ et $w_{\mathrm{Q}}\left(\mathfrak{J}_{1}\right)=w_{\mathrm{Q}}\left(\mathfrak{J}_{2}\right)$.

Ces conditions nous ont permis de tracer les courbes $\theta_{2}(\theta, \tau)=$ Cte (Fig. 6) aussi bien lorsque $\mathfrak{J}_{1}$ est en supraconducteur que lorsqu'il est en cuivre. Nous avons aussi déterminé les limites du domaine d'utilisation des transferts inductifs à haut rendement; elles sont liées à celles de l'interrupteur $\mathfrak{J}_{2}$, à savoir $\theta=\beta \tau$ d'une part et $\mathcal{R}_{\mathrm{e}}$ de l'autre. La première apparaît clairement sur la figure 4 dans le seul cas pratique ( $\mathfrak{J}_{1}$ en cuivre). La deuxième qui précise les temps $\tau$ les plus courts atteignables a été appelée $\mathcal{R}_{\mathrm{T}}$ lorsque $\mathfrak{J}_{1}$ est en cuivre et $\mathcal{R}_{\mathrm{TS}}$ lorsque $\mathfrak{J}_{1}$ est en métal supraconducteur; nous voyons que le domaine d'utilisation de l'ensemble « $\mathfrak{J}_{1}+$ T. I. H. R. $+\mathfrak{J}_{2}$ » est légèrement étendu vers les temps $\tau$ courts comparé au domaine des deux interrupteurs $\mathfrak{J}_{a}$ et $\mathfrak{J}_{b}$ en parallèle.

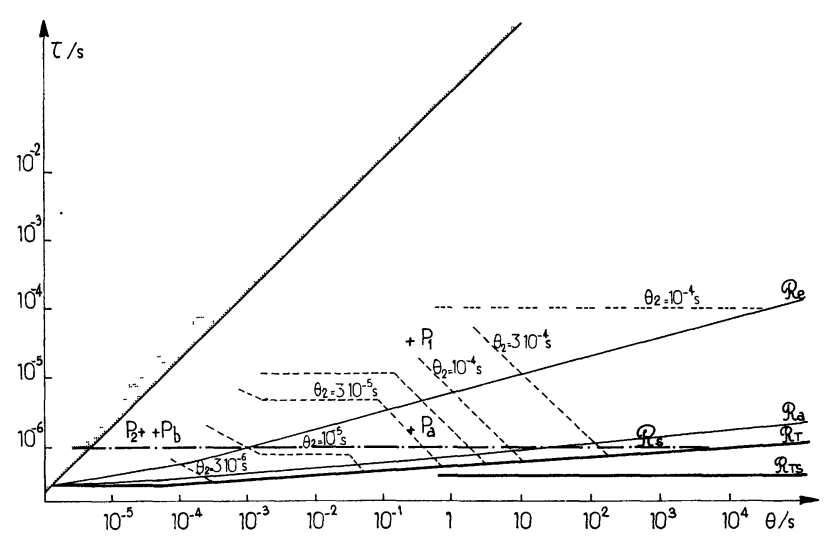

FIG. 6. - Caractéristiques du système « $J_{1}$, T. I. H. R., $J_{2}$ ». On a supposé que le temps de transfert de l'énergie pour $J_{1}$ était égal au temps de latence de $\mathfrak{J}_{2}\left(\tau=\theta_{2}\right)$. En portant le point $P(\theta) \tau$, sur ce graphique, on peut lire la valeur de $\theta_{2}$ lorsque $\mathfrak{J}_{1}$ et $\mathfrak{J}_{2}$ sont tous les deux en cuivre $(--)$ ou lorsque $\mathfrak{J}_{1}$ est en supraconducteur et $J_{2}$ en cuivre $(------)$.

Nous sommes donc maintenant en mesure de déterminer très facilement à partir du point $\mathrm{P}_{\mathrm{a}}$ de coordonnées $(\theta, \tau)$ les points représentatifs de $\mathfrak{J}_{1}$ et $\mathfrak{J}_{2}$ à savoir $\mathrm{P}_{1}\left(\theta, \tau_{1}=\theta_{2}\right)$ et $\mathrm{P}_{2}\left(\theta_{2}, \tau\right)$. Considérons alors le cas où $P$ est situé entre $\mathcal{R}_{\mathrm{e}}$ et $\mathcal{R}_{\mathrm{a}}$. Dans ce cas le transfert peut être réalisé soit par le système

$$
\text { « } \mathfrak{J}_{1}+\text { T. I. H. R. }+\mathfrak{J}_{2} »
$$

soit par l'ensemble de deux interrupteurs en parallèle $J_{a}$ et $\mathfrak{J}_{b}$ représentés par les points $P_{a}$ et $P_{b}$. Chacun de ces deux ensembles comportent deux interrupteurs ayant certaines caractéristiques identiques. Pour $J_{a}$ et $J_{1}$, le temps $\theta$ de passage initial du courant est le même. Mais le temps de transfert d'énergie $\tau$ de $J_{a}$ est beaucoup plus court que le temps de transfert $\tau_{1}$ de $J_{1}$. Il en résulte que dans le cas où $J_{a}$ et $J_{1}$ sont formés du même métal que la bobine de stockage initial, la densité d'énergie transférable par $\mathfrak{J}_{1}$ est très supérieure à celle transférable par $\mathfrak{J}_{\mathrm{a}} ; \mathfrak{J}_{\mathrm{a}}$ sera alors beaucoup plus gros que $J_{1}$. Dans le cas particulier où $\theta<1 \mathrm{~s}$ et $\tau$ est assez court, nous avions indiqué qu'il pouvait être préférable que $J_{a}$ soit en supraconducteur bien que la bobine de stockage soit en cuivre; l'utilisation du T. I. H. R. permet que $\mathfrak{J}_{1}$ soit en cuivre, $\mathfrak{J}_{1}$ restant encore plus petit que $\mathfrak{J}_{\mathbf{a}}$ mais pas de façon aussi sensible. En ce qui concerne $\mathfrak{J}_{2}$ et $\mathfrak{J}_{\mathrm{b}}$, ils sont toujours en cuivre, et $\tau$ est le même pour les deux. Etant donné que nous supposons $P_{\mathrm{a}}$ situé entre $\mathcal{R}_{\mathrm{e}}$ et $\mathcal{R}_{\mathrm{a}}, \theta_{2}$ et $\theta_{\mathrm{b}}$ sont assez peu différents (voir Fig. 6) : les points $P_{2}$ et $P_{b}$ sont proches. $\left(P_{b}\right.$ a été choisi de telle sorte que la densité d'énergie transférable par $\mathfrak{J}_{b}$ soit aussi grande que possible.) Les caractéristiques 
de $J_{2}$ et $J_{b}$ sont donc assez voisines. Nous voyons ainsi que la mise en œuvre du T. I. H. R. permet de faciliter considérablement la réalisation du premier interrupteur dans les systèmes comportant deux interrupteurs en parallèle.

En ce qui concerne les points de fonctionnement situés juste au-dessus de $\mathcal{R}_{\mathrm{e}}$ (pour lesquels nous avions montré au paragraphe précédent qu'il était préférable d'utiliser un seul interrupteur en cuivre plutôt que deux en parallèle), nous pouvons maintenant nous demander s'il ne serait pas bon d'employer aussi un transfert inductif. Comme $w_{\mathrm{Q}}$ est déjà assez grand $\left(10^{11}<w_{\mathrm{Q}}<10^{12} \mathrm{~J} / \mathrm{m}^{3}\right)$, la réponse dépendra de considérations expérimentales annexes.

Le diagramme final montrant les diverses zones d'utilisation des interrupteurs et du transfert inductif est donné figure 7 . Il est bien évident que les limites entre ces diverses zones ne sont qu'approximatives.

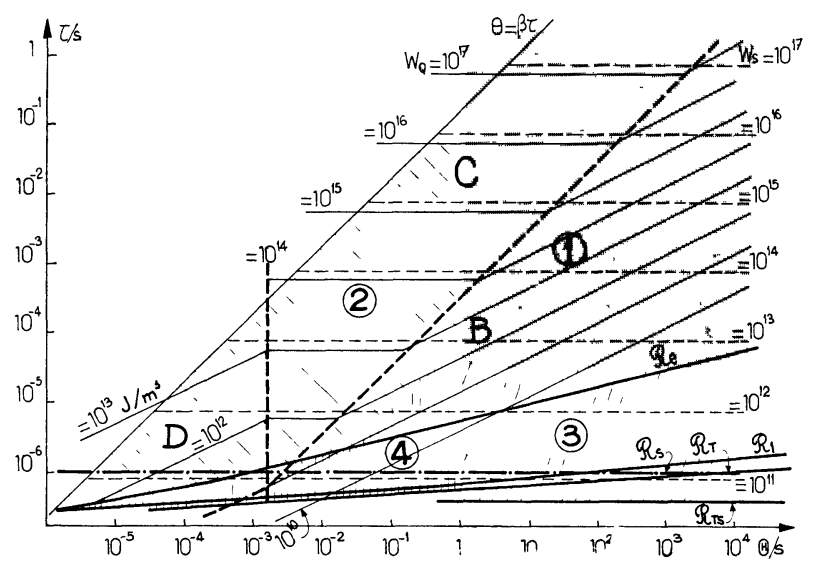

Fig. 7. - Zones d'utilisation des différents systèmes. Dans chacune des zones, le système suivant est le meilleur : zone (1) : interrupteur en supraconducteur unique. Zone (2) : interrupteur en cuivre unique. Zone (3) $: J_{1}$ en supraconducteur, T. I. H. R., $\mathfrak{J}_{2}$ en cuivre. Zone (4) : $\mathfrak{J}_{1}$ en cuivre, T. I. H. R., $\mathfrak{J}_{2}$ en cuivre.

5. Interrupteur à transition supraconducteur-conducteur normal. - Rappelons brièvement le principe de fonctionnement de ces interrupteurs. Pendant la durée $\theta$ du stockage d'énergie qui est généralement effectué par une bobine supraconductrice, l'interrupteur supraconducteur fermé est traversé par un courant de densité $i$. Au moment voulu, on applique brusquement une induction magnétique $B$ qui fait passer le métal de l'état supraconducteur à l'état conducteur normal; sa résistivité devient $\rho_{\mathrm{n}} \cdot\left(\rho_{\mathrm{n}}\right.$ est au plus de l'ordre de $4 \times 10^{-7} \Omega$-m [2].) Il apparaît alors un champ électrique $E_{\mathrm{c}}=\rho_{\mathrm{n}} i$ dans l'interrupteur et la tension $U$ ainsi générée transfère l'énergie vers la charge en un temps $\tau$. Remarquons que des disjoncteurs aux caractéristiques très équivalentes s'obtiennent en réalisant la transition par élévation de la température du conducteur [5].

Considérons maintenant les performances d'un tel interrupteur à transition que nous appelons $J_{T}$ et comparons-les à celles d'un interrupteur à déplace- ment de supraconducteur $\mathfrak{J}_{\mathrm{D}}$. La densité d'énergie transférable par $\mathfrak{J}_{\mathrm{T}}$ est

$$
w_{\mathrm{T}} \simeq E_{\mathrm{c}} i \tau=\rho_{\mathrm{n}} i^{2} \tau .
$$

Elle est très faible. En effet, $E_{\mathrm{c}}$ est le champ électrique qui règne dans un conducteur normal dont la résistivité n'est pas très élevée ; cette valeur est très faible par rapport au champ électrique $E$ supportable par un isolant. Si $w_{\mathbf{D}}$ est la densité d'énergie transférable par $D$, nous avons :

$$
\frac{w_{\mathrm{T}}}{w_{\mathrm{D}}}=\frac{\rho_{\mathrm{n}} i}{E} .
$$

En prenant pour $i$ la valeur maximum de $3,9 \times 10^{9} \mathrm{~A} / \mathrm{m}^{2}$ [2] et avec $E=3 \times 10^{7} \mathrm{~V} / \mathrm{m}$ nous trouvons $w_{\mathrm{T}} / w_{\mathrm{D}} \simeq 5,2 \times 10^{-5}$.

Le volume de supraconducteur à mettre en jeu pour transférer une énergie donnée est au moins 20000 fois plus grand dans l'interrupteur $\mathfrak{J}_{\mathrm{T}}$ à transition supraconducteur-conducteur normal que dans l'interrupteur $\mathfrak{J}_{D}$ à déplacement de supraconducteur. Etant donné le volume à mettre en œuvre, on est en général amené à effectuer la transition sur l'ensemble de la bobine de stockage.

En ce qui concerne l'énergie de commande de $\mathfrak{J}_{\mathrm{T}}$, il faut tout d'abord remarquer que le volume occupé par l'induction magnétique $B$ sera très nettement supérieur d'un facteur $\gamma_{\mathrm{T}}$ à celui du supraconducteur proprement dit. Aussi, l'énergie de commande par unité de volume de supraconducteur s'écrira :

$$
w_{\mathrm{RT}}=\gamma_{\mathrm{T}} \frac{B^{2}}{2 \mu_{0}} \text {. }
$$

Comparons avec la quantité correspondante :

$$
w_{\mathrm{RD}}=\frac{1}{2} \gamma \bar{\omega} v^{2}=\frac{\gamma \bar{\omega} l^{2}}{8 \beta^{2} \tau^{2}}
$$

pour l'interrupteur $\mathfrak{J}_{\mathrm{D}}$. En admettant que les ordres de grandeur des facteurs $\gamma$ et $\gamma_{\mathrm{T}}$ sont les mêmes pour $\mathfrak{J}_{\mathrm{D}}$ et $\mathfrak{J}_{\mathrm{T}}$, il vient :

$$
\frac{w_{\mathrm{RT}}}{w_{\mathrm{TD}}}=\frac{4 B^{2} \beta^{2} \tau^{2}}{\mu_{0} \bar{\omega} l^{2}} .
$$

Par ailleurs, nous avons montré que $J_{D}$ n'était employé seul que pour $\tau \geqslant 30 \mu$ s. Dans ce cas, avec les valeurs typiques $B=1 \mathrm{~T}, \beta=5, l=1 \mathrm{~mm}$, nous déduisons :

$$
\frac{w_{\mathrm{RT}}}{w_{\mathrm{RD}}} \geqslant 7 \text {. }
$$

Nous voyons alors que deux facteurs se conjuguent pour que la mise en œuvre de $J_{D}$ demande beaucoup moins d'énergie que celle de $J_{T}$ : la densité d'énergie de rupture et surtout le volume sont plus petits pour $\mathfrak{J}_{\mathrm{D}}$ que pour $\mathfrak{J}_{\mathbf{T}}$.

Si $\tau<30 \mu$ s, nous avons vu qu'il était préférable d'utiliser un ensemble « $\mathfrak{J}_{1}$ en métal supraconducteur, 
T. I. H. R., $\mathfrak{J}_{2}$ en cuivre » plutôt que $\mathfrak{J}_{\mathrm{D}}$ seul. Le volume de l'interrupteur $\mathfrak{J}_{1}$ est alors le même que celui de $\mathfrak{J}_{2}$ et il est très inférieur à celui qu'aurait $\mathfrak{J}_{\mathrm{D}}$. L'essentiel de l'énergie de commande est dépensé dans $J_{2}$ car la vitesse de déplacement du morceau de cuivre est élevée. On peut alors voir facilement que l'énergie de rupture mise en jeu pour le fonctionnement de l'ensemble est encore incomparablement plus petite que celle mise en jeu pour $\mathfrak{J}_{\mathrm{T}}$.

Enfin, examinons la perte d'énergie due à la chaleur dissipée dans l'appareillage cryogénique par le supraconducteur devenu conducteur normal et effectuant le transfert d'énergie. L'énergie noble à fournir pour évacuer cette chaleur dégagée dans un milieu à très basse température dépasse largement 100 fois la chaleur elle-même. Or, cette dernière représente l'énergie absorbée par l'interrupteur. Dans le cas où la charge est essentiellement inductive, elle est du même ordre de grandeur que l'énergie transférée. La perte est alors considérable.

Pour éviter ce dégagement de chaleur, une solution classique consiste à mettre en parallèle de l'interrupteur à transition supraconducteur-conducteur normal $\mathfrak{J}_{\mathrm{T}}$ une résistance de protection $R_{\mathrm{p}}$ [2] [5] telle que $R_{\mathrm{p}}=R_{\mathrm{n}} / a\left(R_{\mathrm{n}}\right.$ est la résistance de $J_{\mathrm{T}}$ à l'état conducteur normal et $a \gg 1$ ). Dans ce cas, après ouverture de $\mathfrak{J}_{\mathrm{T}}$ l'ensemble $J_{\mathrm{T}}+R_{\mathrm{p}}$ a une résistance égale à $R_{\mathrm{n}} /(1+a) \simeq R_{\mathrm{n}} / a$. Tout se passe au point de vue de la densité d'énergie transférable comme si $\rho_{\mathrm{n}}$ était devenu $\rho_{\mathrm{n}} / a$. Le transfert d'une énergie donnée se fera donc en un temps $a$ fois plus long (ou nécessitera un volume $a$ fois plus grand si l'on désire que le temps de transfert reste le même).

Signalons que si $\tau$ est long, on peut effectuer la transition supraconducteur-conducteur normal dans une partie seulement de $\mathfrak{J}_{T}$; la transition se propage alors à partir de cet endroit. Ainsi, on peut diminuer l'énergie de commande à mettre en jeu. Cependant, la vitesse de propagation de la transition n'est pas très rapide [5] et on ne peut dans ce cas espérer obtenir des temps de transfert d'énergie nettement inférieurs à la ms. Par ailleurs, la technique qui consiste à mettre une résistance de protection en parallèle ne peut plus être aussi efficace et les pertes dans l'appareillage cryogénique deviennent plus importantes.

En conclusion, nous pouvons dire qu'un interrupteur à déplacement de supraconducteur aura toujours un volume incomparablement plus faible qu'un interrupteur à transition supraconducteur-conducteur normal et que l'énergie mise en jeu pour le fonctionnement sera beaucoup plus petite. L'intérêt des interrupteurs à déplacement de supraconducteur est donc manifeste.

6. Conclusion générale. - Pour transférer en un temps $\tau$ sur une charge d'utilisation l'énergie qui a été stockée pendant un temps $\theta$ dans une bobine, on peut utiliser différents dispositifs seuls ou en association (interrupteurs divers, transferts inductifs à haut rendement). Nous avons montré que le choix du système à employer était déterminé par les valeurs de $(\theta, \tau)$ et nous avons indiqué le choix à effectuer sur la figure 7. Si la bobine de stockage est en cuivre, l'interrupteur initial est à déplacement de métal (cuivre ou aluminium qui sont très équivalents). Si la bobine est supraconductrice, l'interrupteur initial est à déplacement de supraconducteur. Cet interrupteur suffit pour effectuer le transfert à moins que $\tau$ ne devienne très court (cf. Fig. 7) ; il faut alors lui associer un système de transfert inductif à haut rendement et un interrupteur à déplacement de métal.

Au cours de notre étude, nous avons aussi vu qu'il était toujours préférable de passer par l'intermédiaire d'un transfert inductif à haut rendement plutôt que de mettre deux interrupteurs simplement en parallèle. Par ailleurs, les interrupteurs à transition supraconducteur-conducteur normal ne paraissent pas compétitifs avec les interrupteurs à déplacement de supraconducteur.

\section{Appendice}

Nous cherchons à déterminer la densité d'énergie transférable $w_{\mathrm{Q}}$ par un interrupteur pour lequel les temps $\theta$ et $\tau$ sont donnés et la valeur maximale $\eta_{M}$ des pertes relatives $\eta$ imposée. Pour cela, nous ferons largement appel aux résultats obtenus dans [1].

Reportons-nous donc à la figure 8 qui est dérivée de la figure 6 de [1] et cherchons les points de fonctionnement $Q$ répondant aux conditions fixées; $w_{\mathrm{Q}} \mathrm{s}$ 'en déduira immédiatement.

Rappelons que ce diagramme représente $\eta(w)$ en

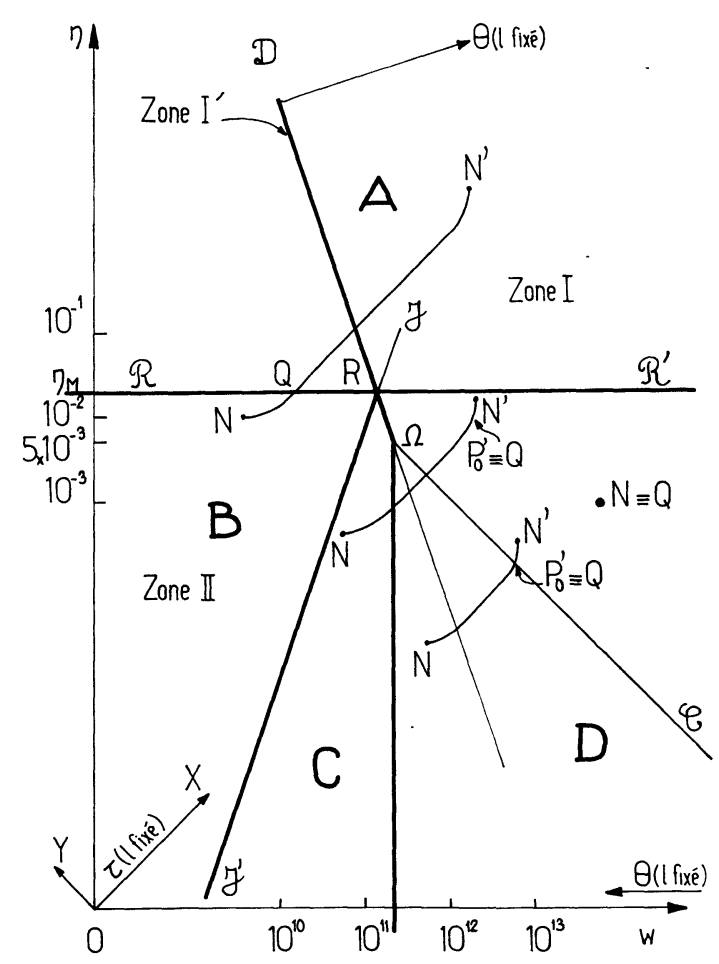

FIG. 8. - Diagramme $\eta(w)$ extrait de [1]. 
coordonnées logarithmiques et que, pour $l$ fixé, il est directement gradué en $\theta$ et $\tau$ (la donnée de $\theta$ et $\tau$ définissant alors le point $\mathrm{N}$ ). Deux zones principales I et II sont à distinguer, séparées par les demi-droites $\Omega \mathcal{D}$ et $\Omega \mathcal{C}$, une troisième zone $\mathrm{I}^{\prime}$ étant la demi-droite $\Omega$ D elle-même. Dans la zone I ainsi que dans la zone $I^{\prime}$, le point $\mathrm{N}$ est le meilleur point de fonctionnement. Dans la zone II, les points de fonctionnement pour $\theta$ et $\tau$ donnés sont à choisir sur un quasi-segment de droite $\mathrm{NN}^{\prime}, \mathrm{N}^{\prime}$ étant construit comme indiqué dans [1]. Nous avons tracé de plus la droite $\mathcal{R} \mathcal{R}^{\prime}$ d'équation $\eta=\eta_{\mathrm{M}}$ qui coupe la droite $\mathcal{D}$ en un point $\mathrm{R}$ ainsi que la droite $\mathcal{J}^{\prime}$ passant par $\mathrm{R}$ et symétrique parallèlement à $O X$ de $\mathcal{R} \mathcal{R}^{\prime}$ par rapport à $\mathcal{D}$. Nous pouvons alors distinguer quatre cas de figure pour déterminer les points de fonctionnement $Q$ cherchés.

Le point $\mathrm{N}(\theta, \tau)$ se trouve dans la zone $A$ au-dessus de $\mathcal{R} \mathcal{R}^{\prime}$ : le problème considéré n'a pas de solution.

Le point $\mathrm{N}$ se trouve dans la zone $\mathrm{B}$ définie par $\mathcal{R} \mathcal{Y}^{\prime}$ : le point de fonctionnement $Q$ cherché est situé alors à l'intersection du quasi-segment $\mathrm{NN}^{\prime}$ et de $\mathcal{R} \mathcal{R}^{\prime}$. On sait que pour un tel point on a $\left(w_{\mathrm{J}}\right)_{\mathrm{Q}} \gg\left(w_{\mathrm{R}}\right)_{\mathrm{Q}}$. On a donc :

$\eta_{\mathrm{M}} \simeq \frac{\left(w_{\mathrm{J}}\right)_{\mathrm{Q}}}{w_{\mathrm{Q}}}=\frac{\rho_{0} i^{2} \theta}{E^{2} i^{2} \tau^{2}} w_{\mathrm{Q}} \quad$ et $\quad w_{\mathrm{Q}}=\eta_{\mathrm{M}} \cdot \frac{E^{2} \tau^{2}}{\rho_{0} \theta}$.

Le point $\mathrm{N}$ se trouve dans la zone $\mathrm{C}$ définie par $\mathrm{R} \mathcal{J}^{\prime}$ et la verticale issue de $\Omega$; notons que le segment $\mathrm{R} \Omega$ (appartenant à $\mathrm{I}^{\prime}$ de [1]) est inclus dans $\mathrm{C}$ lorsque $\eta_{\mathrm{M}}>\eta_{\Omega}$ ce qui sera souvent réalisé puisque $\eta_{\Omega} \simeq 5 \times 10^{-3}$. Le quasi-segment $\mathrm{NN}^{\prime}$ est alors situé (de même que $R \Omega$ ) entièrement en dessous de $\mathcal{R} \mathcal{R}^{\prime}$, le point de fonctionnement $\mathrm{Q}$ est confondu avec $P_{0}^{\prime}$ (ou avec $\mathrm{N}$ sur $\mathrm{R} \Omega$ ). La densité d'énergie transférable est alors déterminée par la densité maximum de courant admissible $i_{\mathrm{M}}^{\mathrm{d}}$ calculée en tenant compte du fait que la chaleur joule dégagée peut diffuser (cf. éq. (47) [1])

$$
w_{\mathrm{Q}}=w_{\mathrm{M}}^{\mathrm{d}}=E i_{\mathrm{M}}^{\mathrm{d}} \tau=\sqrt{\frac{1,45 E^{2} k w_{0}}{\rho_{0} \bar{\omega} c}} \frac{2 \tau}{l} .
$$

Le point $\mathrm{N}$ se trouve dans la zone $\mathrm{D}$, complémentaire des trois autres. Alors, tous les points de fonctionnement $P$ possibles vérifient $\eta_{\mathrm{P}}<\eta_{\mathrm{M}}$. Le point de fonctionnement $Q$ cherché est confondu avec $\mathrm{P}_{0}^{\prime}$ si $\mathrm{N}$ est en dessous de la droite $\mathrm{C}$ et confondu avec $\mathrm{N}$ lorsque $\mathrm{N}$ est au-dessus de cette droite. Dans ces deux cas, le point $\mathrm{Q}$ correspond à la densité maximum de courant admissible lorsque la chaleur joule dégagée ne peut pas diffuser. On a donc :

$$
w_{\mathrm{Q}}^{2}=E^{2} i^{2} \tau^{2}=E^{2} \tau^{2} \frac{w_{0}}{\rho_{0} \theta}
$$

Nous avons porté sur la figure 2 les courbes $w_{\mathrm{Q}}(\theta, \tau)=$ Cte définies par les éq. (1), (2) et (3). Ces trois équations font intervenir la nature de l'isolant et celle du conducteur. De plus, l'éq. (2) (zone C) dépend de $l$ et l'éq. (1) (zone B) dépend de $\eta_{M}$. Ainsi, dans les zones $\mathrm{C}$ et $\mathrm{D}, w_{\mathrm{Q}}$ est indépendant de $\eta_{\mathrm{M}}$ et l'on a $\eta_{\mathrm{Q}}<\eta_{\mathrm{M}}$, tandis que dans la zone $\mathrm{B}$, $w_{\mathrm{Q}}$ est proportionnel à $\eta_{\mathrm{M}}$ et l'on a $\eta_{\mathrm{Q}}=\eta_{\mathrm{M}}$. La transition entre les zones $\mathrm{C}$ et $\mathrm{D}$ a lieu pour $\theta=\theta_{\Omega}$; la longueur de diffusion vaut alors $L_{\Omega}=0,4 l$. La limite $R$ entre les zones $\mathrm{B}, \mathrm{C}$ et $\mathrm{D}$ et la zone $\mathrm{A}$, indiquée par la droite $\mathcal{R} \mathcal{R}^{\prime}$ sur la figure 8 , a été aussi tracée sur la figure 2 . Pour tous les points de fonctionnement $\left(\theta, \tau, \eta_{\mathrm{M}}\right)$ situés sur cette limite, $\tau$ est le temps de transfert le plus court atteignable lorsque $\theta, \eta_{M}$ et $l$ sont donnés. La courbe $\mathcal{R}$ limitant la zone $\mathrm{A}$ représente donc les expressions (55), (57) et (58) de [1] et est par suite l'une des courbes de la figure 12 de [1]. Elle dépend non seulement de la nature de l'isolant, de celle du conducteur et de $\eta_{\mathrm{M}}$ mais aussi de $l$ et $\sqrt{\gamma / \beta^{2}}$. Comme le temps de transfert d'énergie $\tau$ est toujours plus court que la durée $\theta$ de l'impulsion initiale de courant, nous avons aussi indiqué la limite $\theta=\tau$.

Ainsi le diagramme de la figure 2 donne la densité d'énergie transférable pour n'importe quel couple de valeurs $(\theta, \tau)$ données, le problème n'ayant de solution que lorsque le point $(\theta, \tau)$ est au-dessus de la limite $\mathcal{R}$.

\section{Bibliographie}

[1] Rioux, C., Rioux-Damidau, F., Rev. Phys. Appl. 9 (1974) 539.

[2] Laquer, H. L., Ribe, F. L., Weldon, D. M., Proceedings of the 1971 intersociety energy conversion engineering conference, Boston, Mass., U. S. A., 3-5 Aug. 1971 (New York U. S. A. Soc. Automotive Engrs 1971), p. 1089.
[3] Rioux, C., Rev. Phys. Appl. (à paraître).

[4] Rioux-Damidau, F., Rapport LF 14 Laboratoire de Physique des Plasmas (Orsay) 1973.

[5] Ameen, D. L. et Wiederhold, P. R., Rev. Sci. Instr. 35 (1964) 733. 